\title{
Nurses' Roles in Patient Care in Tertiary Level Hospitals in Bangladesh
}

\author{
Rozario M D*, Adhikary H, Gazi HR, Begum D \\ Department of Graduate Nursing, Bangabandhu Sheikh Mujib Medical University, Dhaka, Bangladesh
}

\begin{abstract}
Background: Nurses are hands-on caregivers that make major healthcare contributions. The study was aimed to assess nurses' roles in providing care to the patients admitted in tertiary level hospitals in Bangladesh.

Methods: This descriptive, cross-sectional study was conducted among the registered nurses, during the period of June 2015 - October 2016 in five tertiary level hospitals located in Dhaka, from government and private sectors. Using convenience sampling technique, 225 registered nurses were selected. Data were collected using a pretested structured self-administered questionnaire.
\end{abstract}

Results: More than $64.0 \%$ of the respondents were in the age group of 31- 50 years and only $5.7 \%$ belonged to oldest age (51-60 years) group. Ninety one percent respondents were female, $64.0 \%$ were Muslim and $81.0 \%$ were married. Sixty seven percent were diploma nurses and $89.0 \%$ worked as senior staff nurse and $52.0 \%$ had working experience between 11-30 years. All the respondents played the role of care provider and administering medication. More than $90.0 \%$ of them have played the role of decision maker, communicator and care coordinator. As educator, manager and supervisor $80.0 \%, 71.0 \%$ and $67.0 \%$ of the respondents respectively carried out their responsibility. Only $44.0 \%$ of the respondents played the role of administrator, and $30.0 \%$ played the role as a patient advocator. Ninety nine percent provided bed side care. Documentation, admission, transfer and discharge related activities and patient teaching activities performed by the nurses were found to be respectively $98.0 \%, 92.0 \%$ and $90.0 \%$. Eighty eight percent made round after taking over charge from previous nurse, $81.0 \%$ did planning for nursing care and $78.0 \%$ communicate and coordinate with others. Only $15.0 \%$ respondents were involved in research and $53.0 \%$ with policy making. About half of the respondents had no idea regarding evidence based health service practice (EBHSP).

Conclusion: Nurses in tertiary level hospitals carry out their assigned roles and responsibilities quite satisfactorily. However, due to lack of facilities and knowledge they rarely take part in research. Almost half of the respondents did not have idea about evidence based practices. Appropriate policy and strategy needs to be undertaken by the competent authority to involve nurses in research field and policy making issues. Nurses should be trained on evidence based nursing practice.

Keywords: Nurses role, Evidence based health service practice, Tertiary level hospital

\section{Introduction}

Nurses are the most numerous and vital professional healthcare providers and their demand for the services continue to escalate, resulting in enduring shortages caused by inadequate supply. They are the healthcare provider most proximal to patient bedside in acute care settings. In addition to physician, admitted patients in hospitals for medical procedures also need the assessment and interventions provided by professional nurses.

*Correspondence: Mabel D Rozario, Department of Graduate Nursing, Bangabandhu Sheikh Mujib Medical University, Dhaka, Bangladesh;

e-mail: mabelghosh@yahoo.com

ORCID: https://orcid.org/0000-0001-7044-5775
Nurses are present in hospitals for 24 hours by shifting duty, 7 days a week, observing, intervening, and reporting changes in patient condition. ${ }^{1}$ Nurses are hands-on caregivers that make major healthcare contributions by assessing, planning, and evaluating patient care needs; delivering medications, assuring patient's comfort and advocating for their needs. ${ }^{2}$

An Australian study identified six roles of nurses in general practice which are: patient care, organizer, quality controller, problem solver, educator, and agent of connectivity. The first three roles were appreciated as nursing strengths by both nurses and doctors, but doctors tended not to 
recognise nurse's educator and problem solver roles within the practice. Only $21.0 \%$ of the clinical activities undertaken by nurses that directly funded through medicare. As an agent of connectivity role of the nurse, uniting the different workers within the practice organization, was particularly notable in small and medium size practices, and might be a key determinant of organizational resilience. ${ }^{3}$

Rapid medical and technological advancements and the changing age structure of the population over the last decades contributed to the rapid increasing demand for health care. While changing character of health care created new demands on equipment and material, health services have also been burdened by higher expectation of the patients about service provider's role including nurses. Traditionally, the role of the nurses was to provide care and comfort to the patients as they carried out specific nursing function, but nowadays nursing roles have expanded; now it includes increased emphasis on health promotion and illness prevention, as well as concern for the client as a whole. At present nurses played organized roles such as care giver, communicator, teacher/client family educator, counselor, decision maker, leader/manager, comforter, rehabilitator, protector and advocator/client advocate and career roles e.g. clinical nurse specialist and nurse practitioner role. ${ }^{4}$

A study identified that hospitalized patients demand a more comprehensive care from health professionals, including mental as well as social aspects, with a view to minimize unnecessary mental health risks, such as stress. Therefore, nursing care should be delivered in a humane and holistic manner and in an integrated approach, more comprehensive and personalised care to its patients, with a view to providing high quality care. ${ }^{5}$

The American Nurses Association established the expectation that nurses recognized responsibility and accountability for the delivery of quality care to their patients. It also described the process of professional scope and standards of practice and responsibilities of all professional registered nurses engaged in nursing practice, regardless of setting. ${ }^{6}$

Nurses can play an important role as innovators in shaping quality of care and patient safety. Successful models can be planned, implemented and sustained with solid and effective data. Nursing research helps to build the scientific foundation for clinical practice, prevent complications, and improve patient outcomes. The Robert Wood Johnson Foundation (RWJF) supported through funding the Interdisciplinary Nursing Quality Research Initiative; a program that links evidence-based nursing care to improved patient outcomes. ${ }^{7}$

Nurses are the "heart and soul" of the hospital. They are in a well position to serve on the frontlines quality improvement since they spend most of the time at the patient's bedside and provide patients' care during hospital stay period. ${ }^{8}$

Healthcare quality in Bangladesh is a subject of intense criticism in recent years. Along with other stake holders, it is also nurse's responsibility to collect and use data to improve their own practice as part of broader efforts to improve care. Considering this issue, effort has been undertaken for conducting this research study by the nurses themselves. In recent years, many studies have been conducted to determine quality care, develop strategies to improve quality nursing care but few studies have been conducted to determine the roles of nurses to provide care. To plan an effective and efficient health services for the people, it is important to determine nurse's roles in delivering care for the health care organizations. So, it was attempted to examine the roles of nurses in tertiary level hospitals in providing care to the patients.

The objective of the study was to assess the nurse's role and responsibility in providing care to the patients admitted in tertiary level hospitals in Bangladesh.

\section{Materials and Methods}

This was a descriptive, cross-sectional study conducted among the registered nurses working in the tertiary level hospitals in Bangladesh during June 2015 to October 2016. Data were collected from three government, one public, one autonomous and one non-government tertiary level hospitals located at Dhaka. The hospitals are: Dhaka Medical College Hospital, Sir Salimullah Medical College Hospital (Mitford Hospital), Shaheed Suhrawardy Medical College \& Hospital, Bangabandhu Sheikh Mujib Medical University and Holy Family Red Crescent Medical College and Hospital. 
Study population was registered nurses educated at the level of diploma or above. Important roles and responsibilities of nurses were examined in the study. Care provider, decision maker, communicator, care coordinator, educator, manager, supervisor, administrator and patient advocacy roles were examined. Responsibilities that were examined are: bed side care, administer medication, documentation, nursing round, admission, transfer, discharge, patient teaching, planning nursing care, communication and coordination and research and policy making activities.

Registered nurses who were available during data collection period and voluntarily agreed to participate in the study were included in the study and who did not voluntarily agree to participate in the study and who were found busy with patient care were excluded from the study.

Forty five registered nurses from each hospital that with a total of 225 nurses were enrolled from five hospitals. The sample size was determined by using the standard formula: $\mathrm{n}=\frac{z^{2}(p \times q)}{d^{2}}$.

The estimated sample size was 196 . To minimize the missing $15.0 \%$ more has been added, and finally 225 were included in the study. In absence of any nursing related studies on our research topic either in Bangladesh or in neighbouring countries like India, Pakistan and Sri Lanka, statistical advice was to select at least 45 respondents from each cluster. This will allow the authors to perform parametric multilevel modeling comparison between sites with a maximum of \pm (plus-minus) 2 standard deviation difference between the sites/clusters. According to objectives, it was performed convenient sampling technique for selecting participant.

For collection of data a self-administered structured questionnaire was developed in Bangla, in which there were 39 questions related to the objectives of the study. Before finalisation, the questionnaire was pretested among a small group of registered nurses for understandability. The purpose of the study was explained to the respondents to understand the objectives of the study, and the questionnaire was also explained to them for filling it up comfortably and properly.

A formal ethical clearance was taken from
Institutional Review Board (IRB) of Bangabandhu Sheikh Mujib Medical University.

Ten data collectors for data collection of whom, two different data collectors were assigned in each area at a time to collect data from five places. All the collected data were checked and edited to ensure consistency and accuracy, and transferred to dummy tables for processing and subsequent analysis using a software package - SPSS. This study results will be utilized in improving the quality of nursing care rendered to the patients admitted in hospitals. As a result, the nursing profession will be benefited from this study and researchers will also be inspired to conducting further studies for improving the efficiency of nursing profession.

\section{Results}

In the present study it was identified to which extent registered nurses in tertiary level hospitals execute their roles in providing patient care and also have examined their responsibilities as a care provider of the patients. It was presented the findings under two broad headings: (i) Respondent's socio-demographic and professional characteristics and (ii) Respondent's roles and responsibilities in providing patient care.

(i) Respondents' socio-demographic and professional characteristics

From Socio-demographic characteristics of the respondents it was revealed that age distribution was more or less similar in different age group except the oldest group, where only 5.7 percent nurses are working. Ninety one percent were female, 64.0 percent were Muslims, 28.0 percent Hindus and rests were Christian. Eighty one percent of the respondents were found to be married.

\begin{tabular}{lc}
\multicolumn{2}{l}{ Table I: Respondent's Socio-Demographic Characteristics } \\
\hline Variables & Number (\%) \\
\hline Age in years & $62(27.5)$ \\
$21-30$ & $73(32.4)$ \\
$31-40$ & $77(34.2)$ \\
$41-50$ & $13(5.7)$ \\
$51+$ & \\
\hline Sex & $205(91)$ \\
Female & $20(9)$ \\
Male & $144(64)$ \\
Muslim & $63(28)$ \\
Hindu & $18(8)$ \\
Christian & \\
Marital status & $182(81)$ \\
Married & $43(19)$ \\
Unmarried &
\end{tabular}


Sixty seven percent respondents were diploma nurses, 23.0 percent were BSc nurses whereas 9 percent had MPH degree and only one percent was MSc nursing degree holder. Fifty two percent respondents had working experience between 1130 years. Working fields of respondents were found to be $42.7 \%, 28 \%, 17.3 \%$ and $12.0 \%$ respectively for medicine, surgery, gynae and orthopaedic departments. Among respondents about $89.0 \%$ were senior staff nurse, about $9.0 \%$ nursing supervisor and less than $2.0 \%$ were found to be nursing officer.

Table II: Professional characteristics of the respondents

\begin{tabular}{lc}
\hline \multicolumn{1}{c}{ Variables } & Number (\%) \\
\hline Qualification & \\
Diploma nursing & $150(67)$ \\
BSc nursing & $51(23)$ \\
MSc nursing & $2(1)$ \\
MPH & $22(9)$ \\
\hline Working experience(years) & \\
$1-10$ & $100(44.4)$ \\
$11-20$ & $73(32.4)$ \\
$21-30$ & $44(19.6)$ \\
$31+$ & $8(3.6)$ \\
\hline Working field & \\
Medicine & $96(42.7)$ \\
Surgery & $63(28)$ \\
Gynae & $39(17.3)$ \\
Orthopedic & $27(12)$ \\
\hline Designation & $201(89.3)$ \\
Senior staff nurse & $20(8.9)$ \\
Nursing supervisor & $4(1.8)$ \\
Nursing officer &
\end{tabular}

(ii) Respondent's roles and responsibilities in providing patient care

The magnitude of the nurses assigned roles carried out for providing patient care was assessed. Hundred percent of the respondents said that they worked as a care provider. More than $90.0 \%$ of them have played the role of decision maker, communicator and care coordinator. As educator, manager and supervisor $80.0 \%, 71.0 \%$ and $67.0 \%$ of the respondents respectively carried out their responsibilities. Only $44.0 \%$ of the respondents said that they played role of administrator, and $30 \%$ said that they played the role as a patient advocator.

Though respondents mentioned that $100 \%$ of them performed their responsibilities (figure 1), as care provider, when category of services under care provider was analyzed it was observed that not all respondents were involved in executing their responsibilities for all category of services (figure 2).

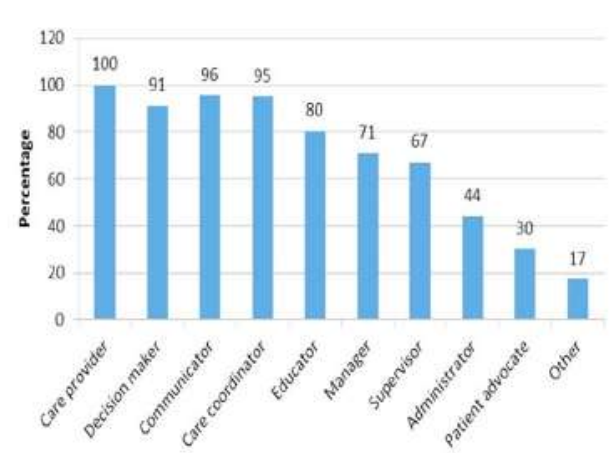

Figure 1: Respondents' Role in Providing Patient Care

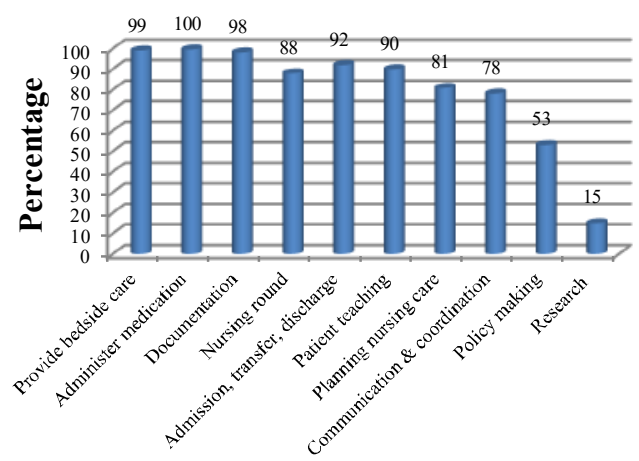

Figure 2: Respondents' Responsibilities as a Care Provider

Ninety nine percent respondents provide bed side care and $100.0 \%$ respondents carried out their responsibility in administering medication. About documentation, admission, transfer and discharge related activities and patient teaching activities respectively $98.0 \%, 92.0 \%$ and $90.0 \%$ respondents replied that they performed these duties Eighty nine percent made round after taking over charge from previous nurse, $81.0 \%$ did planning for nursing care and $78.0 \%$ communicate and coordinate with others. Only $15.0 \%$ respondents were involved in research and $53.0 \%$ with policy making. About half of the respondents had no idea regarding evidence based health service practice (EBHSP).

In bedside care responsibility, they performed multiple tasks such as preparing bed, maintain personal hygiene, observe patient conditions, administer medication, assist to meet need and also show proper respect to patient during providing care. Likewise, for ensuring quality medication, the respondents follow five rights (right patient, right drug, right dose, right route and right time) of medication, direct administration of medicine, assess patient condition, explain the effects, 
observe the effects, take action for side effects and properly document all the records of medication.

The respondents also said that they maintain documentation on admission, transfer, discharge, treatment, investigation, surgery, instrument and supply. According to respondent's statement, eighty nine percent of nurses made their round in the ward after taking over charge from the previous shift, however, about $64.0 \%$ also made round before taking over charge. About $85.0 \%$ of respondents said that they also make round with superintendent and $83.0 \%$ with accompanying all other nurses of the ward.

During admission of patient, as indicators of quality service they prepare bed, receive and assess patient, provide emergency care, inform the doctor and administer medication. During transfer important activities they perform were: obtain written order form doctor, inform patient and family, communicate with staff of concern ward, help the patient to arrange thing and keep transfer records. Likewise, during discharge of patients, respondent obtain written order from doctor, inform patient and family, and explain the prescription, give health teaching to patient and family and advice to attend follow up.

Respondents told that they make patient care plan based on nursing assessment, doctor's prescription, prioritizing need, consulting with others and on available resources.

Regarding communication, respondents mentioned that they communicate with patient with related issues like ward and hospital policy, medical and nursing diagnosis, treatment and prognosis of diseases. Respondents communicate with doctors on new admission, complication, treatment, pathological report and occurrence of any incidence in the ward. They also communicate with their colleagues on issues like care to the patient, treatment and condition of patients, caring plan and nursing diagnosis, working environment and duty roster. For providing quality care, respondents also make effective communication with other departments like administration, dietary section, pathology, pharmacy, radiology, social welfare, laundry and housekeeping.

Only $15.0 \%$ respondents were involved in research and $53.0 \%$ with policy making. Minimum involvement in research is due to time constraint, work overload, lack of experience and interest, financial constraint and over and above due to lack of experts in nursing department in research field. About $50.0 \%$ of the respondents had no idea about evidence based health service practice (EBHSP).

\section{Discussion}

In the present study, it was tried to explore the roles that are played by the nurses in tertiary level hospitals in the country to provide care of patients. The demographic picture of the study population reveals that age distribution in different age category was more or less similar except the oldest age group where it was below 6 percent. Most were female (91.0\%) and Muslims (64.0\%) and $81.0 \%$ were married. This pattern of distribution goes in line with the national pattern of demographic distribution of nurses. Sixty seven percent were diploma holders and most of others were BSc nurse $(23.0 \%)$, more than $50.0 \%$ belonged to 11-30 years of service duration. Therefore, it can be inferred that selection of respondents was appropriate for getting relevant information to examine the study objective.

For providing patient care in hospitals, nurses have to work as care provider, decision maker, communicator, coordinator, educator, manager, supervisor, administrator and patient advocator ${ }^{4}$. Our study showed that nurses of tertiary level hospitals in our country performed many of these roles quite satisfactorily to provide care of patients in the hospitals. It is inspiring that nurses in tertiary hospitals play exceedingly satisfactory role as care provider, decision maker, communicator and coordinator and satisfactory role as educator, manager and supervisor. But their role in administration and patient advocacy needs to be improved (figure 1). A study by Christine B Philips et al showed six roles of nurses in general practice: patient care, organizer, quality controller, problem solver, educator and agent of connectivity which is almost similar to the roles of nurses in this study. But in that study doctors tended not to recognize nurses educator role within the practice. ${ }^{3}$ Whereas in our study $80.0 \%$ nurses said that they performed educator role during their practice. However, doctors perception regarding the role was not explored in this study. 
The administration of medication is a major aspect of patient safety. In this study $97.0 \%$ nurses followed five rights during medication administration which is an indicator of quality nursing care. A similar study in Ethiopia by Hadgu Gerensea et al also found that nurses administer medicine for almost all patients $(96.5 \%)$ using the 5 rights. ${ }^{9}$ In that same study patient observation by the nurses was only $30.0 \%$, but in our study it was $90.0 \%$ which is excellent performance. Besides, in our study during medication more than 90.0\% nurses carried out other responsibilities (asses patient condition, explain effects, provide direct administration, action take for side effects) and $80.0 \%$ nurses maintained documentation.

Hendrich Ann, Skierczynski and Zhenqiang in a study showed that nurses played their roles on patient care, documentation, medication administration, co-ordination, patient assessment and reading vital signs. ${ }^{10}$ Nurses roles in their study were almost similar as in this study. In their study they also examined the time spent for each role played by the nurses which was not done in this study.

Involvement in policy making is an important determinant for ensuring quality care services to the patients but from this study it was revealed that $53 \%$ nurses were involved in policy making issues (like patient care, ward management, patient treatment and supervision). Nurses should make commitment for their contribution in policy making issues and authority should come forward to inspire them to contribute more in policy making matters. Fifty one percent respondents said that they practice Evidence Based Health Service care. This study finding is also discouraging which needs to be addressed sincerely.

Susan Hassmiller identified, nine challenges that individual nurses and nursing profession must address. One of which is generate evidence and engage in research. In elaborating the challenge author mentioned that nursing research helps build the scientific foundation for clinical practice, prevention, and improved patient outcomes ${ }^{7}$. In this study however, only $15.0 \%$ nurses were involved in research. Lack of experts, lack of experience, dearth of interest, time constraint, work overload and financial constraints are the major reasons for non-involvement in research mentioned by the respondents. To overcome these constraints, appropriate strategy needs to be undertaken by the concerned authority.

The study has some limitations. Because of time constraints self-administered questionnaire were used. So, the findings might be inflated by information biases. If it could use observation method, biasness might be minimized. Another limitation was that as the study was conducted in tertiary level hospitals, the findings may not be generalized to all health care settings. Data were collected from the registered nurses only. If it authors would be able to collect the data from other stakeholders like patients, physicians, hospital executives, cross checking could be made and the result might become more reliable.

\section{Conclusion}

The study findings revealed that in tertiary level hospitals nurses carry out their roles and responsibilities with quite satisfaction except research and policy making issues. They have also less experience in practicing evidence base health care service. Therefore, it is recommend for arranging appropriate training on evidence based health service and also for allocating adequate budget by government, public and private universities and nongovernment hospitals to inspire nursing department for conducting research in nursing field. Furthermore, it is suggested for more involvement of nurses in policy making matters as well. To validate this study and to make it generalize, it needs to conduct further study involving different stakeholders (administrators, doctors, patients, relatives etc.) and including different category of hospitals. It is advised to interpret the study results with caution due to certain limitations of the study.

\section{Acknowledgement}

We greatfully acknowledge the research grant received from the Bangabandhu Sheikh Mujib Medical University (BSMMU), Dhaka, Bangladesh. We are greatly thankful to Pro Vice Chancellor, Research and Development; Dean, Faculty of Nursing and to Dean, Faculty of Preventive and Social Medicine and Chairman of Department of Public Health and Informatics 
(DPHI), BSMMU for their continuous support, assistance, and advice to make the study possible. We are also thankful to the participants who shared their opinions and perceptions, and for their time to provide information.

\section{References}

1. Burhans LM. What is good nursing care? The lived meaning of quality nursing care for practicing nurses. The Faculty of the college of nursing, East Carolina University, USA. May 2008.

2. Burhans LM, Alligood MR. Quality nursing care in the words of nurses, journal of advanced nursing, East Carolina University College of Nursing, Greenville, NC, USA. March 2010.

3. Phillips CB, Pearce C, Hall S, Kljakovic M, Sibbald B, Dwan K et al. Enhancing care, improving quality: the six roles of the general practice nurse. Medical Journal of Australia. 2009; 191:92-7.

4. Basavanthapa BT. Fundamental of Nursing. Second Edition. 2002. p 46-47.

5. Freitas JS, Silva AEBC, Minamisava R, Bezerra ALQ,
Sousa MRG. Quality of nursing care and satisfaction of patient attended at a teaching hospital. Rev. LatinoAm. Enfermagem. 2014; 22:454-60.

DOI: doi.org/10.1590/0104-1169.3241.2437

6. Nursing: Scope and Standards of Practice. $2^{\text {nd }}$ Edition, American Nurses Association Silver Spring, Maryland; 2010. p 34-37.

7. Hassmiller S. Nursing's role in health care reform, American Nurse Today, September 2010; 5:68-69.

8. Debra A. Draper, Laurie E. Felland, Liebhaber A, Melichar L. The Role of Nurses in Hospital Quality Improvement. Research Brief, Health System Change. March 2008.

9. Gerensea H, Solomon K, Birhane M, Medhin BG, Mariam TH, Guesh $\mathrm{H}$ et al. Quality of Nursing Care among in- Patient of Medical- Surgical Ward in Axum St. Marry Hospital, Tigray, Ethiopia. 2015; 4 DOI: doi.org/10.4172/2329-6674.1000132

10. Hendrich A, Skierczynski, Lu Z. A 36-Hospital Time and Motion Study: How Do Medical-Surgical Nurses Spend Their Time, Perm J. 2008. DOI:doi.org/10.7812/TPP/08-021 